\section{Première observation d'une épidémie de septicémie hémorragique due à Pasteurella multocida sérotype B6 au Nord-Cameroun}
A. Martrenchar ${ }^{1 *}$
B.M. Njanpop ${ }^{1}$

\begin{abstract}
MARTRENCHAR (A.), NJANPOP (B.M.). Première observation d'une épidémie de septicémie hémorragique due à Pasteurella multocida sérotype B6 au Nord-Cameroun. Revue Elev. Méd. vét. pays trop., 1994. $47(1): 19-20$
\end{abstract}

Pour la première fois au Cameroun, une souche de Pasteurella multocida de sérotype B6 a été isolée d'un foyer de septicémie hémorragique sur des zébus danıs la région de Maga (Province de l'Extrême-Nord du pays). Par une épreuve sur souris, il a été montré qu'il n'y avait pas de protection croisée entre cette souche et une souche de Pasteurella multocida de type E6 utilisćc pour produirc le vaccin contre la pasteurcliose bovine au Cameroun. L'utilisation d'un vaccin mixte incluant les sérotypes E6 et B6 est donc recommandée pour les pays d'Afrique centrale et d'Afrique de l'Ouest.

Mots-clés : Bovin - Zébu - Septicémie hémorragique - Pasteurella multocida - Vaccin - Cameroun.

\section{Introduction}

Les agents étiologiques de la septicémie hémorragique bovo-bubaline sont les sérotypes B6 et E6 de Pasteurella multocida. Le sérotype $\mathrm{B} 6$ est habituellement présent dans les pays asiatiques et les pays d'Afrique de l'Est (Égypte, Ethiopie, Soudan) et le sérotype E6 dans les autres pays africains $(2,3,6)$. D'un point de vue pratique, ceci détermine la valence vaccinale à utiliser par les différents pays. La présente note rapporte le premier isolement de Pasteurella multocida sérotype B6 dans le Nord-Cameroun.

\section{Matériel et méthodes}

En octobre 1992, une équipe du Laboratoire national vétérinaire de Boklé (LANAVET) fut appelée dans la région de Maga (Extrême-Nord du Cameroun) en raison de mortalités importantes sur des zébus. Sur la base d'un diagnostic clinique, des séances de vaccination d'urgence avaient eu lieu contre le charbon symptomatique dans un village et contre le charbon bactéridien dans un autre. On pouvait estimer les mortalités locales à un minimum de 300 têtes au cours du dernier mois. On a prélevé sur les animaux vivants du sang sur EDTA et des exsudats musculaires et la rate sur les animaux morts. Ces prélèvements furent ensemencés sur gélose au sang. L'identification des souches a été réalisée par les techniques bactériologiques classiques et des gale-

* Adresse actuelle : Laboratoire national d'appui au développement agricole, BP 1839, Abidjan, Côte-d'Ivoire.

1. Laboratoire national vétérinaire de Boklé, BP 503, Garoua, Cameroun.

Reçu le 29.7.1993, accepté le 27.1.1994. ries API 20 NE (bioMérieux, Marcy-l'Etoile, France). Le sérotypage des souches de Pasteurella multocida a été réalisé par la méthode d'hémagglutination indirecte (1). L'épreuve de protection sur souris fut réalisée comme décrit ci-après. Vingt-quatre souris furent vaccinées deux fois à 14 jours d'intervalle à l'aide d'un vaccin inactivé contre la septicémie hémorragique produit au LANAVET (PASTOVAX ${ }^{\circledR}$ ). Ce vaccin est préparé à partir de la souche "Makary 89" de Pasteurella multocida de type E6 isolée dans l'Extrême-Nord du Cameroun en 1989. Les souris furent ensuite réparties en 4 lots de 6 animaux chacun et testées en injection sous-cutanée 10 jours après la deuxième injection vaccinale comme indiqué au tableau I, deux lots de souris non vaccinées servant de témoins. Les souches d'épreuve avaient été stabilisées par lyophilisation et conservées à $-20^{\circ} \mathrm{C}$.

TABIEAU I Méthode et résultats du test de protection sur souris.

\begin{tabular}{|l|c|c|c|c|c|c|}
\hline \multicolumn{1}{|c|}{ Numéro du lot } & 1 & 2 & 3 & 4 & 5 & 6 \\
\hline $\begin{array}{l}\text { Nombre de souris } \\
\begin{array}{l}\text { Vaccination } \\
\text { au Pastovax }\end{array}\end{array}$ & 6 & 6 & 6 & 6 & 6 & 6 \\
$\begin{array}{l}\text { Souche d'épreuve } \\
\text { oui }\end{array}$ & oui 89 & oui & oui & non & non \\
$\begin{array}{l}\text { Dose d'épreuve } \\
10^{4}\end{array}$ & $\begin{array}{c}10^{3} \\
\text { DL50 } 92\end{array}$ & $\begin{array}{c}10^{4} \\
\text { DL50 } 92\end{array}$ & $\begin{array}{c}10^{3} \\
\text { DL50 }\end{array}$ & $\begin{array}{c}10^{3} \\
\text { DL50 }\end{array}$ & $\begin{array}{c}10^{3} \\
\text { DL50 }\end{array}$ \\
$\begin{array}{l}\text { Nombre de } \\
\text { Survivants } \\
\text { après 5 jours }\end{array}$ & 4 & 4 & 0 & 0 & 0 & 0 \\
\hline
\end{tabular}

M 89 = Souche de Pasteurella multocida "Makary $89^{\prime \prime} ; 992=$ Souche de Pasteurella multocida "Goulmoun 92".

\section{Résultats}

Au cours de la visite de terrain, les signes cliniques observés sur les bovins étaient une fièvre élevée et une tuméfaction chaude au point d'inoculation du vaccin. A l'autopsie, on notait une congestion généralisée de la carcasse. A partir des différents prélèvements effectués dans les deux villages, une souche de Pasteurella multocida de type B6, dénommée "Goulmoun 92", fut isolée à l'état pur. Les résultats de l'épreuve de protection sur souris sont indiqués dans le tableau I. La souche fut réisolée à partir du sang du coeur prélevé sur tous les animaux morts. Ainsi, la DL50 pour les souris vaccinées était augmentée d'un facteur supérieur à 10000 pour les souris inoculées avec la souche "Makary 89", mais inférieur à 1000 pour les souris inoculées avec la souche "Goulmoun 92". 


\section{Discussion}

Les séances de vaccination dans les troupeaux atteints de septicémie hémorragique présentent le risque de contaminer les animaux sains, à partir des animaux malades, par l'aiguille du manipulateur. La tuméfaction chaude apparaissant alors au point d'inoculation est probablement une réaction locale à l'injection d'un matériel très virulent. De plus, cela pose un problème de diagnostic différentiel avec la forme externe du charbon bactéridien. Dans la région visitée, de graves problèmes d'ordre ethno-sociologique ont aggravé la situation : les éleveurs soupçonnaient le vaccinateur d'avoir volontairement rendus malades leurs animaux. Les autres signes cliniques n'étaient pas spécifiques de la septicémie hémorragique : on n'observait ni d'oedème de la gorge, ni d'hémorragies sous-cutanées à l'autopsie.

A la connaissance des auteurs, c'est la première fois que le sérotype B6 est isolé à l'ouest du Soudan. II pourrait avoir été introduit par des troupeaux en provenance d'Afrique de l'Est (transhumance, commerce, etc.). Les normes internationales (4) admettent une élévation minimale de la DL50 chez des souris vaccinées d'un facteur de 10000 par rapport à des souris non vaccinées. Pour le PASTOVAX ${ }^{\circledR}$, l'estimation effectuée ici permet d'affirmer que ceci est vérifié avec la souche d'épreuve "Makary 89", mais non avec la souche "Goulmoun 92", ce qui oblige à rajouter cette dernière valence dans la préparation vaccinale destinée au Cameroun.

\section{Conclusion}

Etant donné, d'une part, que de nombreux mouvements d'animaux non contrôlés ont lieu à partir du Tchad et du Cameroun vers les autres pays d'Afrique centrale et les pays d'Afrique de l'Ouest et, d'autre part, que les porteurs sains jouent un rôle important dans la diffusion de la maladie (5), il est fortement recommandé que tous les pays d'Afrique centrale et de l'Ouest utilisent un vaccin mixte contenant les deux sérotypes B6 et E6 de Pasteurella multocida. Dans tous les cas, il serait souhaitable que chaque pays africain enregistrant des cas de septicémie hémorragique vérifie que la souche vaccinale qu'il utilise protège contre les souches sauvages présentes sur son territoire.

\section{Remerciements}

Le sérotypage des souches de Pasteurella multocida a été effectué par le laboratoire "PATHOTROP" du CIRADEMVT à Maisons-Alfort, France.

\section{Bibliographie}

1. CARTER (G. R.). Studies on Pasteurella multocida. I. A hemagglutination test for the identification of serological types. Am. J. vet. Res., 1955, 16: $481-484$.

2. DE ALWIS (M.C.L.). Haemorragic septicaemia in cattle and buffaloes. Revue sci. tech. Off. int. Epizoot., 1984, 3 : 707-730.

3. LEFEVRE (P.C.). Atlas des maladies infectieuses des ruminants. Maisons-Alfort, CIRAD-IEMVT, 1991. p. 56-57.

4. Office International des Epizooties. Manual of standards for diagnostic tests and vaccines. Paris, OIE, 1992. p. 313-321.

5. PERREAU (P.). La septicémie hémorragique des bovidés dans le Centre-Afrique. Utilisation d'un vaccin formolé précipité par l'alun. Revue Élev. Méd. vét. Pays Trop., 1960, 13 (1) : 27-42.

6. PERREAU (P.). Contribution à l'étude immunologique de Pasteurella multocida. Existence d'un nouveau type agent de la septicémie hémorragique des bovidés africains. Revue Elev. Méd. vét. Pays Trop., 1961, 14 (3) : 245-256.

MARTRENCHAR (A.), NJANPOP (B.M.). First observation of an outbreak of haemorrhagic septicaemia due to Pasteurella multocida serotype B6 in Northern Cameroon. Revue Élev. Méd. vét. Pays trop., 1994, 47 (1) : $19-20$

For the first time in Cameroon, a strain of Pasteurella multocida scrotype B6 was isolated from a haemorrhagic septicaemia outbreak in Zebu cattle in the area of Maga (Far Northern Province). Through the mouse protection test, evidence was given that there was no cross protection bet ween this strain and the strain of Pasteurella multocida serotype E6 which is used as an inactivated vaccine in Cameroon. Hence, it is recommended to use a combined vaccine including both serotypes for the Central and West African countries.

Key-words : Cattle - Zebu cattle -Haemorrhagic septicaemia - Pasteurella multocida - Vaccine - Cameroon. 